\title{
Correction to: Long-term clinical outcomes and follow-up status in Japanese patients with familial adenomatous polyposis after radical surgery: a descriptive, retrospective cohort study from a single institute
}

\author{
Akihito Babaya $^{1} \cdot$ Tomoki Yamano $^{1}$ (1) $\cdot$ Takaaki Matsubara $^{1} \cdot$ Yuya Takenaka $^{1} \cdot$ Jihyung Song $^{1} \cdot$ Kei Kimura $^{1}$. \\ Michiko Yasuhara ${ }^{1} \cdot K$ Kozo Kataoka ${ }^{1} \cdot$ Naohito Beppu $^{1} \cdot$ Motoi Uchino $^{2} \cdot$ Masataka Ikeda $^{1} \cdot$ Hiroki Ikeuchi $^{2}$. \\ Nagahide Matsubara ${ }^{3} \cdot$ Kazuo Tamura $^{4} \cdot$ Naohiro Tomita $^{1}$
}

Accepted: 29 October 2020 / Published online: 10 November 2020

(C) Springer-Verlag GmbH Germany, part of Springer Nature 2020

Correction to: International Journal of Colorectal Disease (2020) 35:675-684

https://doi.org/10.1007/s00384-020-03524-y

In Table 4 of the original publication, an error occurred where two values were switched inadvertedly. The Wexner score for duration after surgery (year) for " 20 and over" should be $\mathbf{5 . 3 2}$ and for "less than 20 " should be $\mathbf{8 . 5 6}$. The corrected table is shown as follows:

The online version of the original article can be found at https://doi.org/ 10.1007/s00384-020-03524-y

Tomoki Yamano

yamanot@hyo-med.ac.jp

1 Division of Lower Gastrointestinal Surgery, Department of Surgery, Hyogo College of Medicine, 1-1 Mukogawa-cho,

Nishinomiya, Hyogo 663-8501, Japan

2 Division of Surgery, Department of Inflammatory Bowel Disease, Hyogo College of Medicine, 1-1 Mukogawa-cho, Nishinomiya, Hyogo 663-8501, Japan

3 Department of Surgery, Amagasaki Chuo Hospital, 1-21-1 Shioe, Amagasaki, Hyogo 660-0808, Japan

4 Department of Life Science, Faculty of Science and Engineering, Kindai University, 3-4-1 Kowakae, Higashiosaka, Osaka 577-8502, Japan 
Table 4 Factors associated with fecal incontinence by univariate analysis

\begin{tabular}{|c|c|c|c|c|c|c|c|}
\hline \multirow[t]{2}{*}{ Factors } & \multirow[t]{2}{*}{ Category } & \multicolumn{2}{|c|}{ Soiling } & \multirow[t]{2}{*}{ Odds ratio } & \multirow[t]{2}{*}{$P^{*}$ value } & \multirow[t]{2}{*}{ Wexner score } & \multirow[t]{2}{*}{$P^{* *}$ value } \\
\hline & & $(-)$ & $(+)$ & & & & \\
\hline Gender & $\begin{array}{l}\text { Male } \\
\text { Female }\end{array}$ & $\begin{array}{l}5 \\
4\end{array}$ & $\begin{array}{l}24 \\
21\end{array}$ & $\begin{array}{l}1 \\
1.1\end{array}$ & 0.9 & $\begin{array}{l}7.28 \\
7.21\end{array}$ & 0.8 \\
\hline Age (year) & $\begin{array}{l}35 \text { and over } \\
\text { less than } 35\end{array}$ & $\begin{array}{l}8 \\
1\end{array}$ & $\begin{array}{l}21 \\
24\end{array}$ & $\begin{array}{l}1 \\
9.1\end{array}$ & 0.02 & $\begin{array}{l}6.34 \\
8.28\end{array}$ & 0.18 \\
\hline Date of operation & $\begin{array}{l}1986 \sim 1999 \\
2000 \sim 2017\end{array}$ & $\begin{array}{l}6 \\
3\end{array}$ & $\begin{array}{l}18 \\
27\end{array}$ & $\begin{array}{l}0.3 \\
1\end{array}$ & 0.14 & $\begin{array}{l}5.92 \\
8.3\end{array}$ & 0.09 \\
\hline Duration after surgery (year) & $\begin{array}{l}20 \text { and over } \\
\text { less than } 20\end{array}$ & $\begin{array}{l}6 \\
3\end{array}$ & $\begin{array}{l}16 \\
29\end{array}$ & $\begin{array}{l}0.28 \\
1\end{array}$ & 0.09 & $\begin{array}{l}5.32 \\
8.56\end{array}$ & 0.02 \\
\hline Final surgical procedure & $\begin{array}{l}\text { IAA } \\
\text { IPAA }\end{array}$ & $\begin{array}{l}0 \\
4\end{array}$ & $\begin{array}{l}3 \\
41\end{array}$ & $\begin{array}{l}8.8 \times 10^{14} \\
3.0 \times 10^{8}\end{array}$ & $\begin{array}{l}0.002 \\
<0.0001\end{array}$ & $\begin{array}{l}9.7 \\
8.0\end{array}$ & $\begin{array}{l}0.02 \\
0.003\end{array}$ \\
\hline & IACA & 1 & 1 & $3.0 \times 10^{7}$ & 0.1 & 1.5 & 0.2 \\
\hline & IRA & 4 & 0 & 1 & - & 0 & - \\
\hline
\end{tabular}

Publisher's note Springer Nature remains neutral with regard to jurisdictional claims in published maps and institutional affiliations. 